\title{
A COMBINATION OF MONTE CARLO AND CLASSICAL METHODS FOR EVALUATING MULTIPLE INTEGRALS
}

\author{
BY SEYMOUR HABER
}

Communicated by E. Isaacson, February 29, 1968

1. Stochastic quadrature formulas. In the simplest "Monte Carlo" scheme for numerically approximating the integral

$$
I=\int_{G_{s}} f(\mathbf{x}) d \mathbf{x}
$$

$\left(G_{s}\right.$ is the closed unit cube in $\left.E^{s}\right), N$ points $\mathrm{x}_{1}, \cdots, \mathrm{x}_{N}$ are chosen at random in $G_{8}$ and the quantity

$$
J_{0}=\frac{1}{N} \sum_{i=1}^{N} f\left(x_{i}\right)
$$

is taken as an estimate of $I$. The error analysis is probabilistic. Regarding the $x_{i}$ as (pairwise) independent random variables uniformly distributed on $G_{s}, J_{0}$ is a random variable with mean $I$; the amount by which it is apt to differ from $I$ is estimated in terms of its standard deviation $\sigma\left(J_{0}\right)$. In general (for $f \in L^{2}\left(G_{s}\right)$ ),

$$
\sigma\left(J_{0}\right)=C_{0}(f) N^{-1 / 2}
$$

and it is usual to consider $3 \sigma$ (or even $2 \sigma$ ) as a reliable upper bound on $|J-I|$.

Let $D_{s}^{n}$ denote the set of real functions $f$ such that

$$
\frac{\partial^{n_{1}+\cdots+n_{s}}}{\left(\partial x^{1}\right)^{n_{1}} \cdots\left(\partial x^{s}\right)_{s}} f\left(x^{1}, x^{2}, \cdots, x^{s}\right)
$$

is continuous on $G_{8}$ whenever $n_{1}, n_{2}, \cdots, n_{s} \leqq n$. N. S. Bahvalov [ 1 ], in a study of lower bounds on quadrature errors showed that for the class $D_{s}^{n}$ the error of any nonrandom (e.g. Newton-Cotes, Gaussian) quadrature method is $\Omega\left(N^{-n / s}\right) ; 1$ for random methods the best he could show was $\sigma=\Omega\left(N^{-(n / s+1 / 2)}\right)$ and he showed that for the set of periodic functions in $D_{s}^{n}$ there in fact exist methods for which $\sigma$ $=O\left(N^{-(n / s+1 / 2)}\right)$.

In this note I shall give a general description of a class of formulas which combine the Monte Carlo and classical approaches to get

${ }^{1}$ Hardy's notation: $f=\Omega(g)$ iff $g=O(f)$. 
errors of the order of $N^{-(n / s+1 / 2)}$ for the class $D_{s}^{n}$, and construct some specific formulas of this class for the case $n=2$. A more complete development, and proofs, will appear elsewhere.

Definition. A "stochastic quadrature formula (s.q.f) of degree $n$ (for $G_{s}$ )" is a set of 1-dimensional random variables $A_{1}, \cdots, A_{k}$ and $s$-dimensional random variables $X_{1}, \cdots, X_{k}$, such that

(1) $\sum_{i=1}^{k} A_{i} P\left(X_{i}\right) \equiv \int_{G_{s}} P$ whenever $P$ is a polynomial (in $s$ variables) of degree $n$ or lower; but there is a polynomial $P^{*}$ of degree $n+1$ such that

$$
\sum_{i=1}^{k} A_{i} P^{*}\left(X_{i}\right) \not \equiv \int_{G_{i}} P^{*}
$$

(2) $m\left(\sum_{i=1}^{k} A_{i} f\left(\boldsymbol{X}_{i}\right)\right)=\int_{G_{s}} f$ whenever $f \in L^{2}\left(G_{s}\right)$ (" $m(\cdot)$ " denotes the mean of a random variable).

For example, $X_{1}$ uniformly distributed over $G_{s}, X_{2}=(1 / 2, \cdots$, $1 / 2)-X_{1}$, and $A_{1} \equiv A_{2} \equiv 1 / 2$ define an s.q.f. of degree 1 .

I shall write " $Q(f)$ " for $\sum_{1}^{k} A_{i} f\left(\boldsymbol{X}_{i}\right)$, and speak of "the quadrature formula $Q . "$ In the usual way one may apply $Q$ to any region $A$ obtainable from $G_{s}$ by an affine transformation, without changing its degree. The adapted formula will be denoted by " $Q_{(A)}$." I shall denote by " $Q_{M}$ " the formula resulting from partitioning $G_{s}$ into $M$ congruent subcubes and applying $Q$ to each. The number of function evaluations used in a quadrature formula will be denoted by " $N$ "; for $Q_{M}, N=k M$.

THEOREM. If $Q$ is a stochastic quadrature formula of degree $n-1$ and $f \in D_{s}^{n}$, then

$$
\sigma\left(Q_{M}(f)\right) \sim C(f) N^{-(n / 8+1 / 2)}
$$

where

$$
C(f)=\frac{k^{n / 8+1 / 2}}{2^{n+8} n !}\left(\sum m_{i j} \int_{G_{s}} f^{(i)} f^{(j)}\right)^{1 / 2} .
$$

Here " $f(N) \sim g(N)$ " means $f(N) / g(N) \rightarrow 1$ as $N \rightarrow \infty$. The sum in (3) runs over all $n$-tuples $i$ and $j$ of integers between 1 and $s$. The notations used are: If $i=\left(i^{1}, i^{2}, \cdots, i^{n}\right), j=\left(j^{1}, \cdots, j^{n}\right)$, then

$$
f^{(i)}=\frac{\partial^{n} f}{\left(\partial x^{i_{1}}\right) \cdot \cdots\left(\partial x^{i_{n}}\right)}, \quad \mathbf{x}^{(i)}=x^{i_{1}} \cdot x^{i_{2}} \ldots \cdot x^{i_{n}}
$$

and

$$
m_{i j}=m\left(\left(Q_{(A)}\left(\mathrm{x}^{i}\right)-\int_{A} \mathrm{x}^{i}\right)\left(Q_{(A)}\left(\mathrm{x}^{j}\right)-\int_{A}\left(\mathrm{x}^{j}\right)\right)\right)
$$


where $A=A_{s}$ is the cube $\left|x^{i}\right| \leqq 1, i=1,2, \cdots, s$.

$C(f)$ will rarely be known a priori; however a good a posteriori estimate of $\sigma\left(Q_{M}(f)\right)$ may be obtained by a modification of the calculation in the manner described in [3].

2. Formulas of degree 2. In [2] an s.q.f. $Q$ of degree zero with $k=1$ was investigated; in [3] one of degree 1 with $k=2$ was given. For $n \geqq 2$ the situation is more complicated; it is a consequence of a theorem of Stroud [4], that

$$
k \geqq\left(\begin{array}{c}
n+s \\
{[n / 2]}
\end{array}\right)
$$

("[ $[$ " denoting the greatest integer function), so that $k$ cannot be independent of $s$. For constant coefficient formulas we have

THEOREM. If

$$
Q(f)=\frac{1}{k} \sum_{i=1}^{k} f\left(X_{i}\right)
$$

is an s.q.f. of degree $\geqq 2$ for $G_{s}$, then $k \geqq 3 s+1$.

THEOREM. If $\left(a_{i, j}\right)$ is a $(3 s+1) \times k$ real matrix such that

(1) $a_{i, j}=k^{-1 / 2}$ for all $j$,

(2) $a_{i, 1}^{2}+a_{i, 2}^{2}+\cdots+a_{i, k}^{2}=1$ for all $i$,

(3) $a_{i, 1} a_{i^{\prime}, 1}+a_{i, 2} a_{i^{\prime}, 2}+\cdots+a_{i, k} a_{i^{\prime}, k}=0$ if $i \neq i^{\prime}$,

(4) $a_{i, j}^{2}+a_{i+1, j}^{2}+a_{i+2, j}^{2}=3 / k$ for all $j$ and for $i=2,5,8, \cdots, 3 s-1$, we shall denote by " $V_{L}$ " $(L=1,2, \cdots, s)$ the subspace of $E^{k}$ spanned by the $(3 L-1) s t, 3 L t h$, and $(3 L+1)$ st rows of $\left(a_{i, j}\right)$ and by " $S_{L}$ " the sphere of radius $(3 / k)^{1 / 2}$ in $V_{L}$, centered at the origin. Then if

$$
X_{j}=\left(X_{j}^{1}, X_{j}^{2}, \cdots, X_{j}^{s}\right), \quad j=1,2, \cdots, k
$$

are random variables such that, for $L=1,2, \cdots s$,

$$
\left(X_{1}^{L}, X_{2}^{L}, \cdots, X_{k}^{L}\right)
$$

is uniformly distributed on $S_{L}$, then

$$
Q(f)=\frac{1}{k} \sum_{j=1}^{k} f\left(X_{j}\right)
$$

is an s.q.f. of degree 2 for the cube $A_{8}$.

It remains to be seen for which $k$ such matrices exist; it is desirable that $k$ be as low as possible. Here we have 
THEOREM. If there exists a Hadamard matrix ([5], [6]) of order $r$, then for any $s$ such that $3 s+1 \leqq r$, there is a $(3 s+1) \times r$ matrix $\left(a_{i, j}\right)$ satisfying the conditions of the above theorem.

For the top row of the Hadamard matrix $H_{r}$ may be taken to have all entries $=1$; and then the first $3 s+1$ rows of $r^{-1 / 2} H_{r}$ satisfy all conditions.

Since Hadamard matrices of order $r=4 p$ are known to exist at least up to $p=29, k$ can be taken $\leqq 3 s+4$ for $s \leqq 38$; and can in fact be taken equal to $3 s+1$ for $s=1,5,9, \cdots, 33$.

The classical approaches to efficient quadrature have been: (1) To take advantage of as much smoothness as the integrand may have by constructing formulas of maximum degree using a fixed number of points; (2) To find formulas with a fixed number of points which minimize the error for functions with a given degree of smoothness. The second seems the more practical approach for functions of several variables, where smoothing is apt to be very difficult. With the present formulas, partitioning $G_{s}$ reduces the error as quickly as possible for each fixed smoothness class $D_{s}^{n}$; while the first approach continues in use, to reduce the number $k$ in (3).

\section{REFERENCES}

1. N. S. Bahvalov, On approximate calculation of multiple integrals, Vestnik Moscow Univ. 4 (1959), 3-18.

2. S. Haber, A modified Monte Carlo quadrature, Math. Comp. 20 (1966), 361-368. 397.

3. —_, A modified Monte Carlo quadrature. II. Math. Comp. 21 (1967), 388-

4. A. H. Stroud, Quadrature methods for functions of more than one variable, Ann. New York Acad. Sci. 86 (1960), 776-791.

5. J. Williamson, Note on Hadamard's determinant theorem, Bull. Amer. Math. Soc. 53 (1947), 608-613.

6. S. W. Golomb and L. D. Baumert, The search for Hadamard matrices, Amer. Math. Monthly 70 (1963), 12-17.

National Bureau of Standards, Washington, D.C. 